\title{
Testing the limb-darkening coefficients measured from eclipsing binaries
}

\author{
A. Claret \\ Instituto de Astrofísica de Andalucía, CSIC, Apartado 3004, 18080 Granada, Spain \\ e-mail: claret@iaa.es
}

Received 9 January 2008 / Accepted 5 February 2008

\section{ABSTRACT}

\begin{abstract}
Aims. The numerical methods used to compute limb-darkening coefficients (LDCs) are still a matter of discussion. To improve this scenario, we have revised our earlier calculations and compare the new LDCs with previous theoretical ones, as well as with empirical values measured from eclipsing binaries.

Methods. We present new LDCs, based on the least-squares method but computed with higher numerical resolution (100 $\mu$ points instead of 11). The LDCs are computed for the following photometric bands: Johnson-Cousins, Strömgren, Geneva, and Walraven systems. Bolometric and monochromatic calculations are also available on request. The computations are presented for 19 metallicities ranging from $10^{-5}$ up to $10^{+1}$ times the solar abundance, with $\log g$ values between 0.0 and 5.0, effective temperatures between $2000 \mathrm{~K}$ and $50000 \mathrm{~K}$, and microturbulent velocities from $0 \mathrm{~km} \mathrm{~s}^{-1}$ to $8 \mathrm{~km} \mathrm{~s}^{-1}$.

Results. The new theoretical LDCs provide better fits to the calculated specific intensities than those derived by adopting the $r$-integration. The improvement is approximately one order of magnitude. When compared with empirical linear LDCs measured for nine eclipsing binaries, it was not possible to distinguish our results from those provided by the $r$-integration method. However, this comparison also reveals that the theoretical atmosphere models are unable to give a satisfactory fit to the observations. This result is supported by a recent investigation of transiting extrasolar planets. These discrepancies may also be related to problems with the empirical LDC values themselves.
\end{abstract}

Key words. stars: binaries: eclipsing - stellar: atmospheres

\section{Introduction}

There is still controversy concerning the best numerical method of computing theoretical limb-darkening coefficients (LDCs) using the predictions of model atmospheres. The least-squares method (LSM) gives a better match to the intensity distributions calculated using model atmospheres $(I(\mu))$, but the resulting fluxes with these fits are not always equal to the computed ones. In the case of the flux conservation method (FCM) the fluxes are, by definition, conserved but the intensity distributions are not described well. By adopting the LSM, we are seeking a compromise between the best fit to $I(\mu)$ and simultaneously keeping the flux-conservation as close as possible to the computed values. If a suitable law is selected, the LDCs based on the LSM represent the function $I(\mu)$ very well, at any part of the stellar disc for any filter or wavelength, $\log g$, effective temperature, metallicity, and microturbulent velocity. For a detailed discussion of the comparison between LSM and FCM, see Díaz-Cordovés et al. (1995) and Claret (2000).

However, even if we consider only the LSM, we find some ambiguity. For example, should the central value of the specific intensity, $I(1)$, be fitted with the intensity profile? If we choose to include it, the resulting fit is in general better because we are adjusting one additional parameter. There are some variants of the classical LSM, and recently Heyrovský (2007; hereafter H07) have presented a numerical method by minimizing the radially integrated, squared residuals. His main conclusion is that such a method produces better fits and better flux conservation. H07 used a set of nine eclipsing binaries, for which empirical linear LDC values are available, to test his new method.

In this paper we revise our calculations of LDCs carried out previously (Claret 2000). We present new LDCs that have been calculated using the LSM, including additional $\mu$ points and fitting for $I(1)$. A critical comparison between the new LDCs and observational results is carried out for the same set of eclipsing binaries used by H07. We also present a new numerical method for computing LDCs, based on a strategy for solving the differential equation of stellar structure, though only in a preliminary form.

\section{The numerical methods}

The laws most commonly used in limb-darkening investigations are the following:

The linear law:

$\frac{I(\mu)}{I(1)}=1-u(1-\mu)$

The quadratic law:

$\frac{I(\mu)}{I(1)}=1-a(1-\mu)-b(1-\mu)^{2}$.

The square root law:

$\frac{I(\mu)}{I(1)}=1-c(1-\mu)-d(1-\sqrt{\mu})$. 
The logarithmic law:

$\frac{I(\mu)}{I(1)}=1-e(1-\mu)-f \mu \ln (\mu)$,

where $I(1)$ is the specific intensity at the centre of the disc, and $u, a, b, c, d, e$, and $f$ are the corresponding LDCs. The quantity $\mu$ is defined by $\mu=\cos (\gamma)$, where $\gamma$ is the angle between the line of sight and the emergent intensity. Claret (2000) introduced a new law that includes a combination of five terms:

$$
\frac{I(\mu)}{I(1)}=1-\sum_{k=1}^{4} a_{k}\left(1-\mu^{\frac{k}{2}}\right) \text {. }
$$

The limb-darkening law described in Eq. (5) has several advantages over the linear or bi-parametric approximations. First, the linear and bi-parametric laws only provide acceptable fittings for limited effective temperature ( $\left.T_{\text {eff }}\right)$ ranges; for other $T_{\text {eff }} \mathrm{s}$, their intrinsic accuracy is poor. The law given in Eq. (5) permits the use of a single law for the whole HR diagram with higher accuracy. Second, it reproduces the intensity distributions obtained using model atmospheres very well. Third, it conserves flux to within very small tolerances. Finally, these advantages do not depend on the physics adopted for model atmosphere calculations.

We computed LDCs using the above limb-darkening laws. The model atmosphere intensities were convolved with a response function that takes into account the terrestrial atmospheric transmission, filter transmission curves, detector sensitivity, and double reflection from an aluminium-coated mirror. The LDCs were computed for 29 filters: Johnson-Cousins $U B V R I J H K$, Strömgren uvby, Geneva $G U G B 1 G B G B 2 G V 1 G V G G$ and Walraven $W W W U W L W B W V$ systems. We denote the Geneva and Walraven filters with the prefices $G$ and $W$ to differentiate them from each other and from the Johnson-Cousins system. All calculations were carried out for airmass 1.0. More details on the response function can be found in Claret (2000, 2003).

The quality of the fits can be evaluated by using the merit function

$\sigma^{2}=\frac{1}{N-M} \sum_{i=1}^{N}\left(y_{i}-Y_{i}\right)^{2}$,

where $y_{i}$ is the model intensity at point $i, Y_{i}$ the fitted function at the same point, $N$ the number of points, and $M$ the number of coefficients to be adjusted. Another criterion taht will be used is the quantity $\Delta F \equiv \frac{\left|F_{a}-F_{a, \mathrm{imb}}\right|}{F_{a}}$, where $F_{a}$ is the actual flux at the passband $a$ and $F_{a \text {,limb }}$ is the flux at the same passband using a given limb-darkening law.

For the model atmosphere calculations, we adopted the ATLAS (Kurucz 2000) and PHOENIX (Hauschildt 2000) models. The LDC calculations were performed for 19 metallicities ranging from $10^{-5}$ up to $10^{+1}$ time the solar abundance, for surface gravities $0.0 \leq \log g \leq 5.0$, and for effective temperatures $2000 \leq \frac{T_{\text {eff }}}{\mathrm{K}} \leq 50000$. Five microturburlent velocity values $\left(V_{\xi}=0,1,2,4,8 \mathrm{~km} \mathrm{~s}^{-1}\right)$ were also considered. The available results are summarized in Table 1 . As mentioned above, there is also a possibility of fitting the central intensity, $I(1)$. We carried out these calculations for the same ranges of parameter values as in Table 1.

\section{Comparison with previous theoretical works}

The comparisons made in this section refer mainly to the recent work by $\mathrm{H} 07$, as we have already made comparisons with other methods. In that paper, two methods were proposed based on the minimisation of the integrated squared residuals. If the variable $\mu$ is selected as the integration variable, the method provides very similar results to those using the FCM. If the radial coordinate $r$ is used (hereafter $r$-integration), H07 argues that it improves the flux conservation and the LDCs agree better with the observational data for a small sample of eclipsing binaries and the Sun (compared to the calculations by Claret 2000).

However, the comparison between the theoretical LDCs by Claret (2000) and H07 were not made with similar numerical conditions. Only $11 \mu$ points were used by Claret (2000) to compute LDCs, whereas approximately $50 \times 10^{6}$ points were used in the $r$-integration by $\mathrm{H} 07$ to characterise the spline coefficients. The LDCs derived using the $r$-integration are also different from those by Claret (2000) concerning the adjustment of $I(1)$ (see above). An interesting feature can be found in the Table 1 given by H07. The $r$-integration method seems to perform better in the case of the poorer limb-darkening laws (e.g. the linear one), but gives practically the same results as Claret (2000) when a more sophisticated law (Eq. (5)) is used, even considering only $11 \mu$ points. Limb darkening is an inherently non-linear phenomenon. Therefore, to search for a optimal linear - or bi-parametric - approximation is not the best approach. The key procedure is also to search for the best function, not only to try to find better fits for simpler approximations.

To verify whether the differences found by $\mathrm{H} 07$ are due to the numerical resolution and to the fitting of $I(1)$, we revisited our own LDC calculations to derive new results for an increased number of $\mu$ points. In these computations we fitted the central intensity, as used in the $r$-integration method, to make the comparison clearer. The ATLAS models are only tabulated for $17 \mu$ points, ranging from 0.01 to 1.0 . We interpolated extra $\mu$ points by using splines.

In Figs. 1 and 2 we can see that increasing the number of $\mu$ points makes an important improvement in the quality of the flux conservation. We plotted the quantity $\Delta F$ (filters $B V R I$ ) as a function of $\log T_{\text {eff }}$ for two cases: $11 \mu$ points and $100 \mu$ points for the laws described by Eqs. (5) and (1), respectively. The mean values of $\Delta F$ for $100 \mu$ points $(\Delta F 100)$ are around one order of magnitude more accurate than that for $11 \mu$ points $(\Delta F 11)$. In addition, the mean values of $\Delta F 100$ (and the maximum deviations considering all filters) are also smaller than the values derived by adopting the $r$-integration for the same laws. Unfortunately, H07 only computed LDCs for the BVRI passbands, so a more complete comparison is not possible.

In this work we have considered some filters ( $U$ and $u$ ) that have in the past yielded LDCs with large $\sigma \mathrm{s}$. The results presented here for these problematic filters have $\sigma \mathrm{s}$ that are lower than those obtained using only $11 \mu$ points. These results are not directly comparable to the definition introduced by H07. Note also that the $\Delta F 100 \mathrm{~s}$ are almost independent of $T_{\text {eff }}$ while there is a strong correlation with $T_{\text {eff }}$ when $11-\mu$-point fitting is adopted.

We have therefore shown that the apparent superiority of the $r$-integration method stems from a higher numerical resolution and the inclusion of $I(1)$ in the fittings. When extended to a closer numerical resolution, our own calculations produce more accurate $\Delta F$ values than the $r$-integration method. 


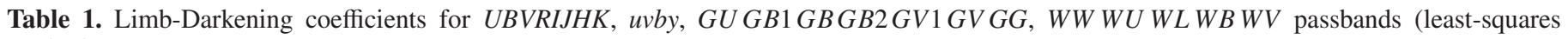
method).

\begin{tabular}{|c|c|c|c|c|c|c|}
\hline Name & Source & $T_{\text {eff }}(\mathrm{K})$ & $\log g$ & $\log [\mathrm{M} / \mathrm{H}]$ & $V_{\xi}\left(\mathrm{km} \mathrm{s}^{-1}\right)$ & Fit type \\
\hline Table 2 & ATLAS & $3500-50000$ & $0.0-5.0$ & -5.0 & 2 & Eq. (5), 24 filters \\
\hline Table 3 & ATLAS & $3500-50000$ & $0.0-5.0$ & -4.5 & 2 & Eq. (5), 24 filters \\
\hline Table 4 & ATLAS & $3500-50000$ & $0.0-5.0$ & -4.0 & 2 & Eq. (5), 24 filters \\
\hline Table 5 & ATLAS & $3500-50000$ & $0.0-5.0$ & -3.5 & 2 & Eq. (5), 24 filters \\
\hline Table 6 & ATLAS & $3500-50000$ & $0.0-5.0$ & -3.0 & 2 & Eq. (5), 24 filters \\
\hline Table 7 & ATLAS & $3500-50000$ & $0.0-5.0$ & -2.5 & 2 & Eq. (5), 24 filters \\
\hline Table 8 & ATLAS & $3500-50000$ & $0.0-5.0$ & -2.0 & 2 & Eq. (5), 24 filters \\
\hline Table 9 & ATLAS & $3500-47500$ & $0.0-5.0$ & -1.5 & 2 & Eq. (5), 24 filters \\
\hline Table 10 & ATLAS & $3500-50000$ & $0.0-5.0$ & -1.0 & 2 & Eq. (5), 24 filters \\
\hline Table 11 & ATLAS & $3500-50000$ & $0.0-5.0$ & -0.5 & 2 & Eq. (5), 24 filters \\
\hline Table 12 & ATLAS & $3500-50000$ & $0.0-5.0$ & -0.3 & 2 & Eq. (5), 24 filters \\
\hline Table 13 & ATLAS & $3500-50000$ & $0.0-5.0$ & -0.2 & 2 & Eq. (5), 24 filters \\
\hline Table 14 & ATLAS & $3500-50000$ & $0.0-5.0$ & -0.1 & 2 & Eq. (5), 24 filters \\
\hline Table 15 & ATLAS & $3500-50000$ & $0.0-5.0$ & 0.0 & 2 & Eq. (5), 24 filters \\
\hline Table 16 & ATLAS & $3500-50000$ & $0.0-5.0$ & +0.1 & 2 & Eq. (5), 24 filters \\
\hline Table 17 & ATLAS & $3500-50000$ & $0.0-5.0$ & +0.2 & 2 & Eq. (5), 24 filters \\
\hline Table 18 & ATLAS & $3500-50000$ & $0.0-5.0$ & +0.3 & 2 & Eq. (5), 24 filters \\
\hline Table 19 & ATLAS & $3500-45000$ & $0.0-5.0$ & +0.5 & 2 & Eq. (5), 24 filters \\
\hline Table 20 & ATLAS & $3500-40000$ & $0.0-5.0$ & +1.0 & 2 & Eq. (5), 24 filters \\
\hline Table 21 & ATLAS & $3500-50000$ & $0.0-5.0$ & 0.0 & 0 & Eq. (5), 24 filters \\
\hline Table 22 & ATLAS & $3500-50000$ & $0.0-5.0$ & 0.0 & 1 & Eq. (5), 24 filters \\
\hline Table 23 & ATLAS & $3500-50000$ & $0.0-5.0$ & 0.0 & 4 & Eq. (5), 24 filters \\
\hline Table 24 & ATLAS & $3500-50000$ & $0.0-5.0$ & 0.0 & 8 & Eq. (5), 24 filters \\
\hline Table 25 (Sun) & ATLAS & 5777 & 4.377 & 0.0 & 1.5 & Eq. (5), 24 filters \\
\hline Table 26 (Vega) & ATLAS & 9400 & 3.90 & -0.5 & 0 & Eq. (5), 24 filters \\
\hline Table 27 & ATLAS & $3500-50000$ & $0.0-5.0$ & all metallicities & $0,1,2,4,8$ & Eq. (1), 24 filters \\
\hline Table 28 & ATLAS & $3500-50000$ & $0.0-5.0$ & all metallicities & $0,1,2,4,8$ & Eq. (2), 24 filters \\
\hline Table 29 & ATLAS & $3500-50000$ & $0.0-5.0$ & all metallicities & $0,1,2,4,8$ & Eq. (3), 24 filters \\
\hline Table 30 & ATLAS & $3500-50000$ & $0.0-5.0$ & all metallicities & $0,1,2,4,8$ & Eq. (4), 24 filters \\
\hline Table 31 & PHOENIX & 2000-9800 & $3.5-5.0$ & 0.0 & 2 & Eq. (5), 24 filters \\
\hline Table 32 & PHOENIX & 2000-9800 & $3.5-5.0$ & 0.0 & 2 & Eq. (1), 24 filters \\
\hline Table 33 & PHOENIX & 2000-9800 & $3.5-5.0$ & 0.0 & 2 & Eq. (2), 24 filters \\
\hline Table 34 & PHOENIX & 2000-9800 & $3.5-5.0$ & 0.0 & 2 & Eq. (3), 24 filters \\
\hline Table 35 & PHOENIX & 2000-9800 & $3.5-5.0$ & 0.0 & 2 & Eq. (4), 24 filters \\
\hline
\end{tabular}

\section{Comparison between theoretical-calculated LDCs}

H07 presented a comparison between his calculated LDCs, those from Claret (2000), and empirical values measured for nine eclipsing binary stars (see H07, his Table 2). The H07 LDCs are systematically smaller than those from Claret (2000), and this difference is greater at longer wavelengths (see H07, his Fig. 5). There are two reasons for this: the inclusion of $I(1)$ as a fitted parameter, and the number of $\mu$ points used by H07. Both of these aspects will tend to decrease the LDCs for the linear law, which is the only law for which a comparison with empirical results is possible. In Fig. 3 we illustrate the effect of increasing the number of $\mu$ points. We return to this point below.

Before we compare the LDCs computed in this work with observations, it is important to consider some aspects of the comparison carried out by H07. Modelling the light curves of eclipsing binaries is a complex task, and limb darkening has much less effect on the light curve shape than do several other parameters. The LDCs can also be correlated with the measured relative radii of the stars. At the level of precision achieved in most eclipsing binary analyses, it is not possible to distinguish one law from another (see the papers by Lacy and collaborators in the reference list), and it is in fact rare to be able to measure LDCs to a useful accuracy even for the linear law. As an example of this, consider the linear LDCs found for the secondary components of EEPeg (Lacy \& Popper 1984) and FS Mon
(Lacy et al. 2000b). These two stars have very similar $T_{\mathrm{eff}} \mathrm{S}$ (around $6500 \mathrm{~K}$ ) and $\log g$ s (round 4.2 ), but the measured $B$ band LDCs are 0.75 for EE Peg B and 0.59 for FS Mon. This difference is much greater than some of the quoted error bars, although in the case of EE Peg B, the quoted error is 0.15. The use of varied observational material and different light curve analysis techniques means that a comparison with theoretical predictions, particularly for the inaccurate linear law, is not straightforward.

In addition to observational uncertainties, it seems that there are also problems with the atmosphere models themselves. The difference between the observed and theoretical prediction for the $V$-band in the case of MU Cas is 0.23 , for example, which is $40 \%$ of the empirical value. This may be evidence of problems intrinsic to the ATLAS models, but also may indicate that the empirical measurements are not accurate enough to allow a reliable comparison with theoretical predictions.

Another point that can influence the comparison between theory and observations is the uncertainty in the $T_{\text {eff }}$ measurements for the eclipsing binaries. It was recently shown that the error bars are optimistic for some eclipsing binaries (Claret $2006,2007 b)$. This problem can be reduced by using the ratio of the temperatures ( $\left.T R \equiv T_{\text {eff2 } 2} / T_{\text {eff } 1}\right)$ instead of the $T_{\text {eff }} \mathrm{s}$ themselves. The ratio $T R$ is much better constrained because it is directly derived from the light curve analysis so is independent of temperature calibrations, distances, and model atmosphere analyses. We evaluated the theoretical error bars due to uncertainties in the $T_{\mathrm{eff}} \mathrm{s}$, metallicities, and $V_{\xi} \mathrm{s}$ (Fig.4). The more 


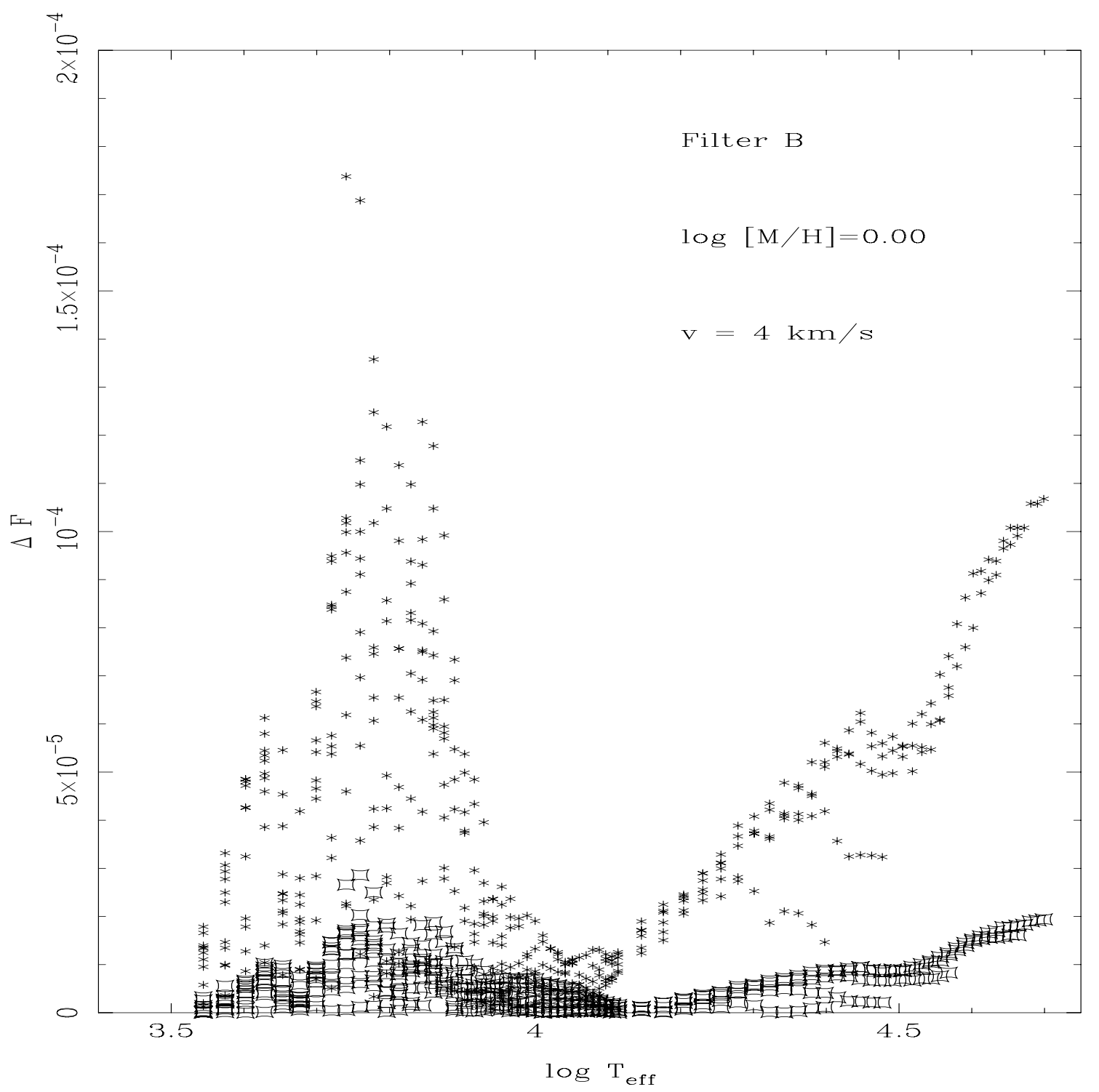

Fig. 1. Relative flux $(\Delta F)$ as a function of $\log T_{\text {eff }}$, for Eq. (5). Asterisks represent 11- $\mu$-point fits, while open squares denote 100- $\mu$-point fits.

important contribution to these errors comes from the uncertain-

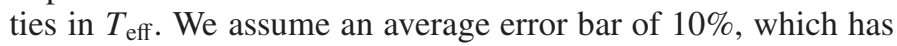
the most effect on the linear LDCs for cooler stars in the sample. The parameter $V_{\xi}$ has practically no effect, while the metallicity ( \pm 0.5 dex) changes the LDCs in a similar way to the $T_{\text {eff }} \mathrm{s}$ but to a lesser extent. $\mathrm{H} 07$ does not consider these sources of uncertainty in his analysis.

The LDCs should also be corrected for the irradiation effect (see Claret 2007a). Although this is a necessary correction, it will equally affect the LDCs presented here and those from $\mathrm{H} 07$. These corrections depend on relative fluxes of the components, and are only marginally important here because the effects of mutual irradiation are minor for the systems listed in Table 2.

Considering the above points, we performed a comparison between our linear LDCs and the H07 sample of eclipsing binaries. The values are given in Table 2 and plotted in Figs. 4 and 5. Unavailable LDCs were set to 0.00. Our first conclusion from this comparison is that theoretical LDCs are unable to satisfactorily match the observational results, no matter which numerical method is adopted. That this can be attributed to the shortcomings in the linear limb-darkening law is supported by recent studies of the bright eclipsing binary $\beta$ Aurigae
(Southworth et al. 2007a) and of several transiting extrasolar planetary systems (Southworth et al. 2007b; Southworth 2008). The disagreement between the theoretical and observed $V$-band LDCs for MU Cas, no matter which numerical method is used, also indicates that the level of accuracy of the observations and/or theoretical atmosphere models does not yet permit a useful comparison.

Independently of the disagreement between theory and observations, the comparison in Fig. 5 shows that we cannot tell whether our theoretical approach is better than that of $\mathrm{H07}$ because the two methods give practically the same results (taking the theoretical and observational error bars into account). This conclusion also holds if we adopt the simpler comparison method used by H07 (which neglects some error sources). However, as shown in Sect. 3, our calculation method with 100 or more $\mu$ points provides better $\Delta F$ s than those by $\mathrm{H} 07$ and therefore should be preferred.

\section{Discussion}

As mentioned above, the main purpose of a limb-darkening law based upon LSM is to provide very small $\Delta F \mathrm{~s}$, as well as small $\sigma$ s. However, the resulting $\sigma$ only gives an average value 


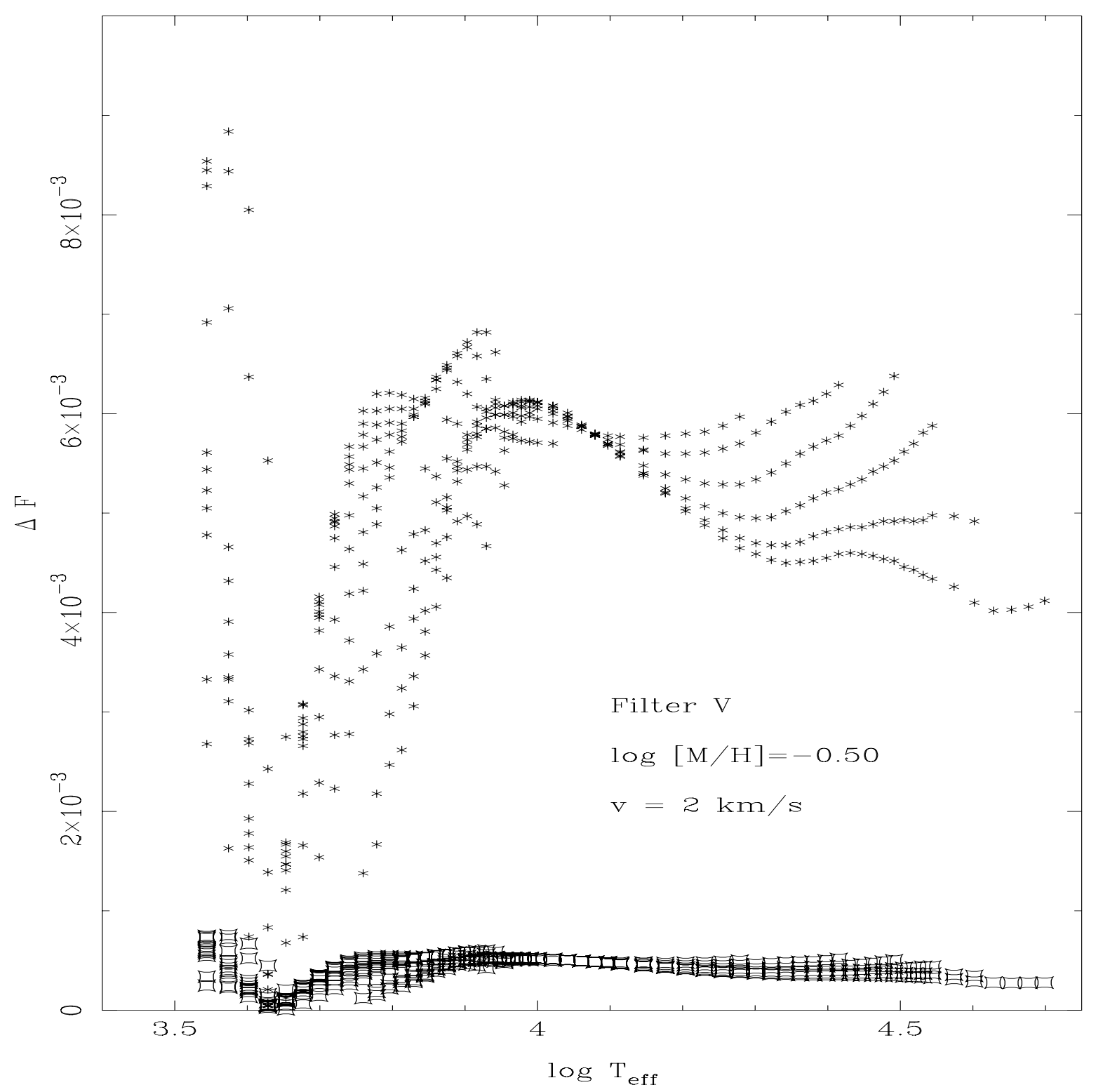

Fig. 2. The same as for Fig. 1 but using the linear limb-darkening law (Eq. (1)).

Table 2. Empirical linear limb-darkening coefficients. Filters $B V R$.

\begin{tabular}{cccccccccc}
\hline \hline Name & $T_{\text {eff1 }}$ & $T_{\text {eff2 }}$ & $\log g_{1}$ & $\log g_{2}$ & $F_{\mathrm{r}}$ & $B_{1,2}$ & $V_{1,2}$ & $R_{1,2}$ \\
\hline BS Dra & 6619 & 6619 & 4.25 & 4.25 & 0.01 & $0.64 \pm 0.04,0.64 \pm 0.04$ & $0.50 \pm 0.03,0.50 \pm 0.03$ & $0.00,0.00$ & 1 \\
EE Peg & 8700 & 6456 & 4.13 & 4.33 & 0.07 & $0.62 \pm 0.03,0.75 \pm 0.15$ & $0.00,0.00$ & $0.00,0.00$ \\
FS Mon & 6715 & 6550 & 4.03 & 4.18 & 0.05 & $0.58,0.594$ & $0.58,0.591$ & 2 \\
GG Ori & 9950 & 9950 & 4.27 & 4.28 & 0.005 & $0.50 \pm 0.04,0.50 \pm 0.04$ & $0.51 \pm 0.03,0.51 \pm 0.03$ & $0.23 \pm 0.07,0.00$ \\
WW Cam & 8350 & 8240 & 4.16 & 4.20 & 0.03 & $0.00,0.00$ & $0.494 \pm 0.017,0.499 \pm 0.017$ & $0.00,0.00$ & 3 \\
V459 Cas & 9140 & 9100 & 4.14 & 4.14 & 0.005 & $0.00,0.00$ & $0.487 \pm 0.008,0.487 \pm 0.008$ & $0.00,0.00$ & 5 \\
MU Cas & 15100 & 14750 & 3.97 & 3.86 & 0.01 & $0.00,0.00$ & $0.56 \pm 0.07,0.56 \pm 0.07$ & $0.00,0.00$ & 7 \\
WW Aur & 7960 & 7670 & 4.16 & 4.16 & 0.03 & $0.616 \pm 0.056,0.512 \pm 0.078$ & $0.416 \pm 0.060,0.418 \pm 0.083$ & 0.000 .00 & 8 \\
RW Lac & 5760 & 5560 & 4.26 & 4.41 & 0.003 & $0.00,0.00$ & $0.55,0.57$ & $0.00,0.00$ & 9 \\
\hline
\end{tabular}

References to Table 2: 1 Popper \& Etzel (1981); 2 Lacy \& Popper (1984); 3 Lacy et al. (2000b); 4 Torres et al. (2000); 5 Lacy et al. (2002); 6 Lacy et al. (2004a); 7 Lacy et al. (2004b); 8 Southworth et al. (2005); 9 Lacy et al. (2005).

for the whole stellar disc. Quite different fits can have very similar $\sigma \mathrm{s}$, arising either from a small difference for large numbers of points or a large difference for only a small number of points. An examination of Fig. 1 presented by H07 shows that the $r$-integration method provides the worst fit near the limb (almost 30\% of the difference) when compared with other limb-darkening fits, including those by Claret with $11 \mu$ points. At other parts of the disc, the $r$-integration method gives better agreement.

We checked the capability of the $100-\mu$-point approach to fit the solar-specific intensities, using the Kurucz (2000) model atmosphere for the Sun. In Fig. 6 we show the behaviour of $\Delta F$ 


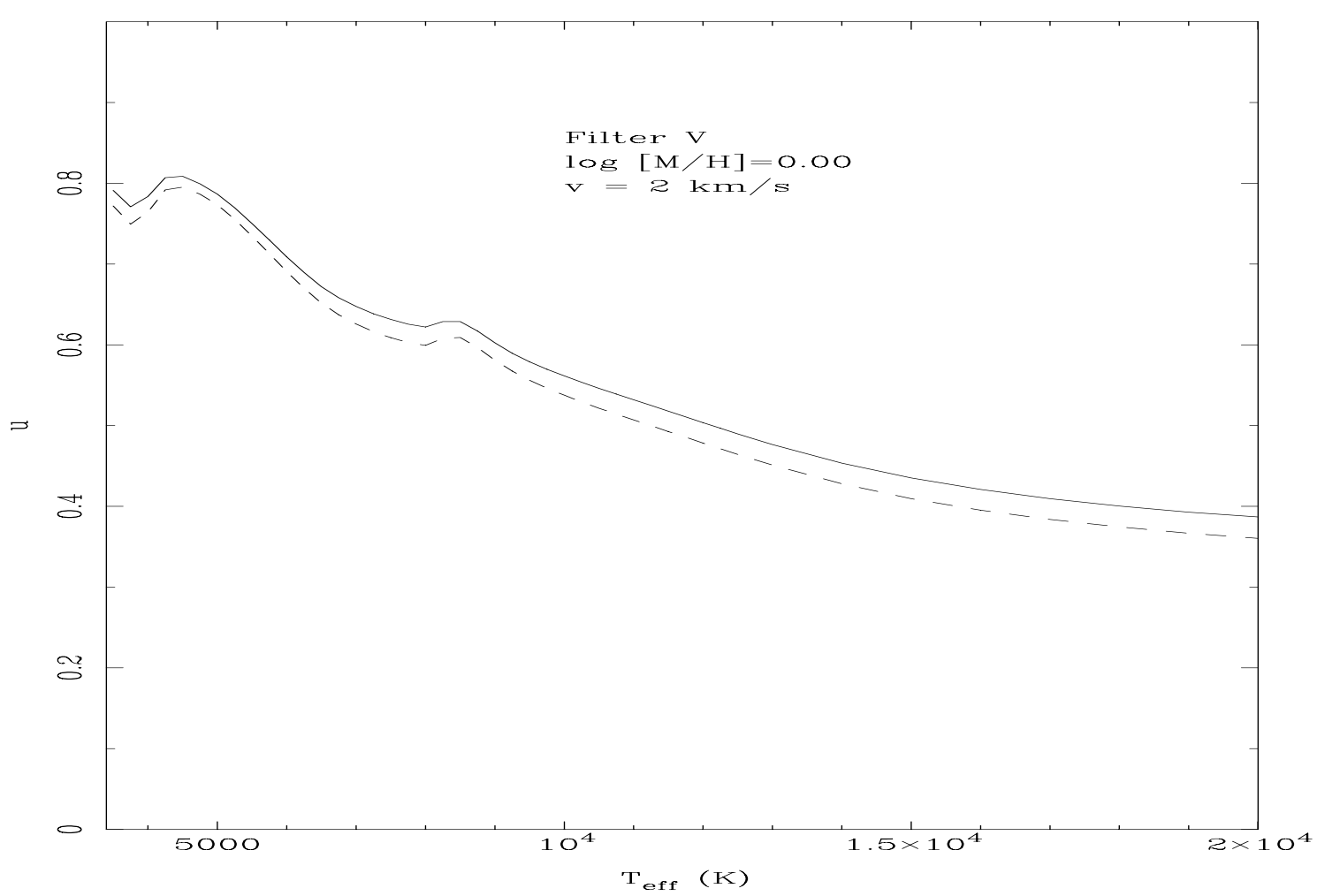

Fig. 3. Linear limb-darkening coefficients computed with 11 (continuous line) and 100 (dashed line) $\mu$ points, as a function of the effective temperature, for the $V$-band.

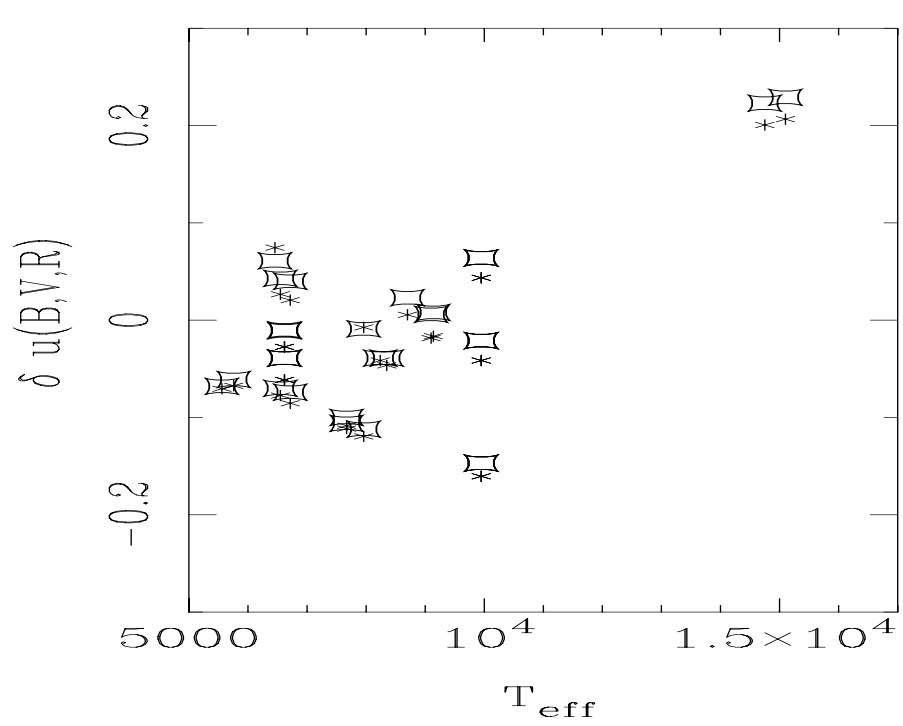

Fig. 4. The theoretical and observed linear LDCs for the nine eclipsing binaries. The open crosses and stars refer to the $B$ and $V$ bands, while full squares denote the $R$ band. Only the theoretical error bars are shown.

as a function of wavelength, with the upper line calculated using the linear law and the lower line using Eq. (5). The latter results are essentially independent of wavelength over the range considered, and have very low $\Delta F$ values of the order of $2 \times 10^{-5}$. The linear fit is much worse, although it remains better than those presented by H07 (his Fig. 6). We note that limb darkening is not a linear phonomenon, and thus recommend the use of Eq. (5).

At this point, it is appropriate to ask if LSM is capable of providing still better fits than those derived by adopting 100 equally spaced $\mu$ points. We now introduce a variant of the LSM that

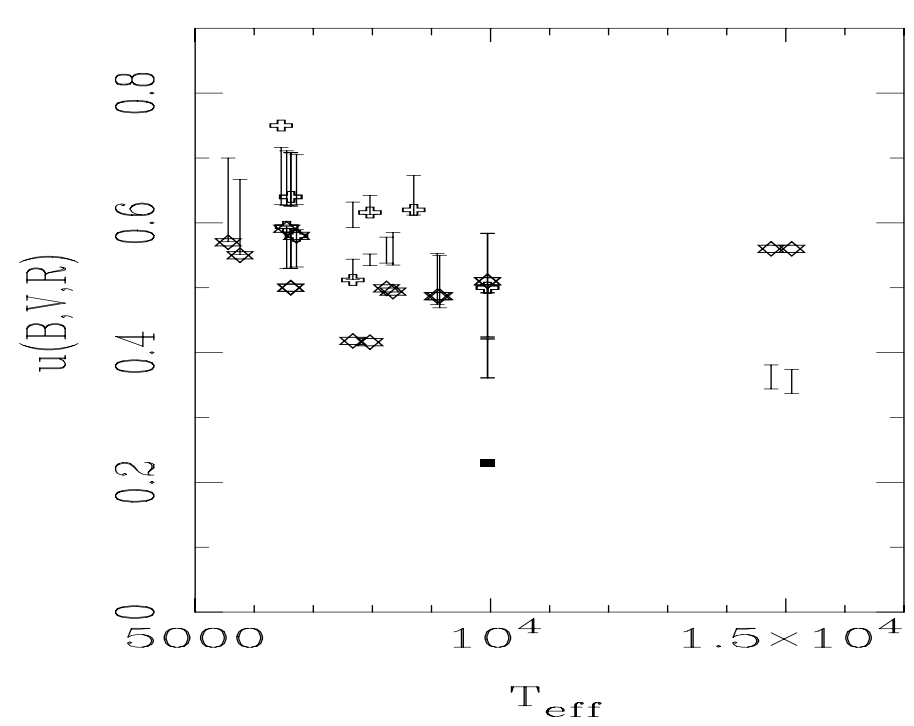

Fig. 5. Differences between the theoretical and observed linear LDCs for the nine eclipsing binaries. Asterisks indicate the results by adopting the present calculations - with $100 \mu$ points and $I(1)=1-$ while open squares denote the calculations by $\mathrm{H} 07$.

is based on strategies for solving the differential equations of stellar structure. First, we apply the standard LSM to $n$ equally spaced points. The quantities $\left|y_{i}-Y_{i}\right|$ are then computed for all passbands, at each $\mu$ point. If this quantity is larger than a given tolerance at a given point, then this point is considered to be an outlier and is not considered in the next iteration. The remaining $\mu$ points are redistributed (some points may be added) and the process repeated until an appropriate accuracy is reached. This method is still being tested, but we can present some preliminary results here. 


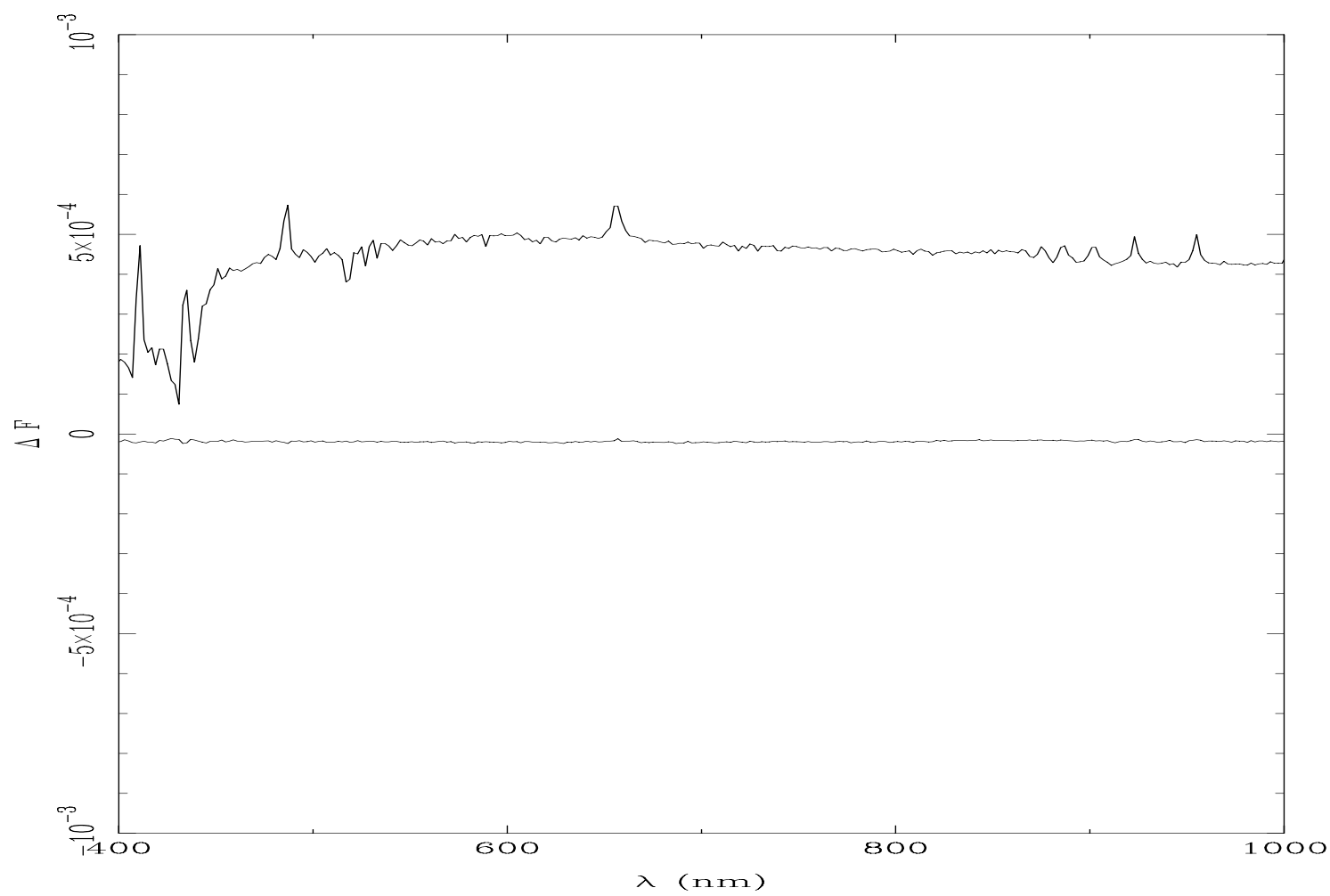

Fig. 6. The upper continuous line represents the fit of the linear law to the specific intensity of the Sun. The lower continuous line shows the fit obtained using Eq. (5).

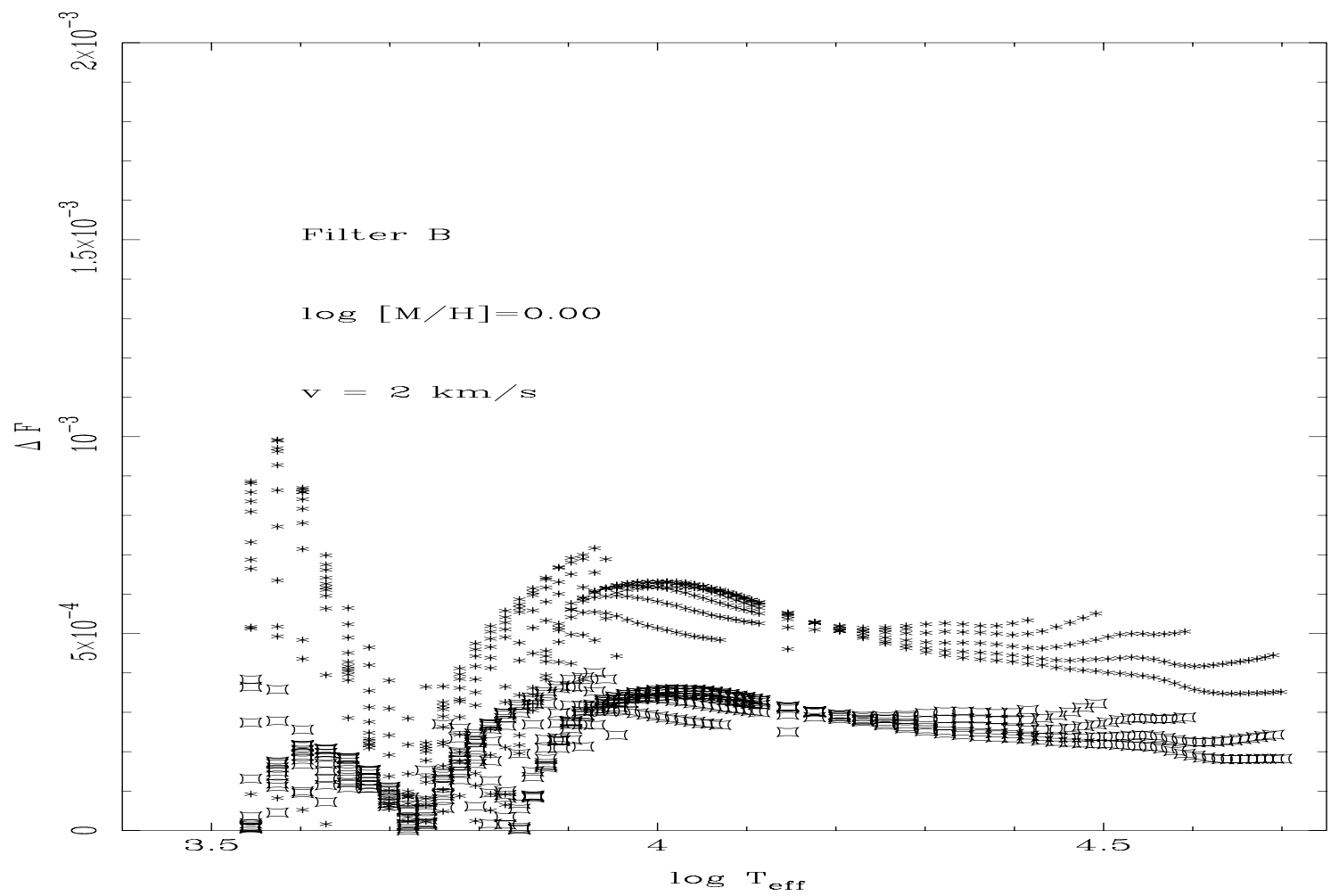

Fig. 7. Relative flux $(\Delta F)$ as a function of $\log T_{\text {eff }}$ for the linear law (Eq. (1)). Asterisks represent the 100- $\mu$-point fits while open squares denote the preliminary calculations using the new method proposed in this work.

In general, the number and the distribution of the $\mu$ points depends on the convolved intensity profile. It seems, at first sight, that the larger the number of $\mu$ points, the better the flux conservation. However, numerical experiments show that an adequate distribution of a few $\mu$ points may be more advantageous than simply adopting a large number of points, if a decent fitting function is selected. The average $\Delta F$ s tabulated by $\mathrm{H} 07$ shows this in the case of Eq. (5): the resulting $\Delta F \mathrm{~s}$ (and his definition of $\sigma$ ) 
obtained using $r$-integration $\left(50 \times 10^{6}\right.$ points) are similar to those derived by adopting standard LSM but using only $11 \mu$ points.

How do the fits using the above method compare with the traditional LSM and with those by H07? Our preliminary calculations provide $\Delta F \mathrm{~s}$ around a factor of two less than those derived using the traditional LSM with $100 \mu$ points. When compared with the results by $\mathrm{H} 07$, the maximum value of $\Delta F$ is around 20 times lower. On the other hand, the mean value of $\Delta F$ is around 10 times lower. We have illustrate these results for the $B$-band in Fig. 7.

Acknowledgements. I am in debt to J. Southworth for his comments/suggestions. The Spanish MEC (AYA2006-06375) is gratefully acknowledged for its support during this work.

\section{References}

Claret, A. 2000, A\&A, 363, 1081

Claret, A. 2003, A\&A, 401, 657

Claret, A. 2006, A\&A, 445, 1061

Claret, A. 2007a, A\&A, 470, 1099

Claret, A. 2007b, A\&A, 475, 1019
Díaz-Cordovés, J., Claret, A., \& Giménez, A. 1995, A\&AS, 110, 329 Hauschildt, P. H. 2000, private communication

Heyrovský, D. 2007, ApJ, 656, 483 (H07)

Kurucz, R. L. 2000, private communication

Lacy, C. H. S., \& Popper, D. M. 1984, ApJ, 281, 268

Lacy, C. H. S., Torres, G., Claret, A., \& Sabby, J. A. 2000a, AJ, 120, 3226

Lacy, C. H. S., Torres, G., Claret, A., et al. 2000b, AJ, 119, 1389

Lacy, C. H. S., Torres, G., Claret, A., \& Sabby, J. A. 2002, AJ, 123, 1013

Lacy, C. H. S., Vaz, L. P. R., Claret, A., \& Sabby, J. A. 2003, AJ, 128, 1324

Lacy, C. H. S., Torres, G., Claret, A., \& Sabby, J. A. 2004a, AJ, 128, 1340

Lacy, C. H. S., Torres, G., Claret, A., \& Sabby, J. A. 2004b, AJ, 128, 1840

Lacy, C. H. S., Torres, G., Claret, A., et al. 2004c, AJ, 128, 3005

Lacy, C. H. S., Torres, G., Claret, A., \& Vaz, L. P. R. 2005, AJ, 130, 2838

Papaloizou, J., \& Pringle, J. E. 1980, MNRAS, 193, 603

Popper, D. M. 1984, AJ, 89, 132

Popper, D. M., \& Etzel, P. B. 1981, AJ, 86, 102

Southworth, J. 2008, MNRAS, submitted

Southworth, J., Maxted, P. F. L., \& Smalley, B. 2004, MNRAS, 351, 1277

Southworth, J. Smalley, B., Maxted, P. F. L., Claret, A., \& Etzel, P. B. 2005, MNRAS, 363, 529

Southworth, J., Bruntt, H., \& Buzasi, D. L. 2007a, A\&A, 467, 1215

Southworth, J., Wheatley, P. J., \& Sams, G. 2007b, MNRAS, 379, L11

Torres, G., Lacy, C. H. S., Torres, G., Claret, A., \& Sabby, J. A. 2000, AJ, 120, 3226 